ARTICLE

DOI: $10.1038 / 541467-017-00809-9 \quad$ OPEN

\title{
Establishing and storing of deterministic quantum entanglement among three distant atomic ensembles
}

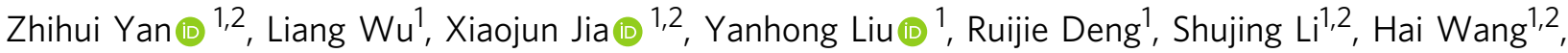 \\ Changde $X_{i e^{1,2}} \&$ Kunchi Peng ${ }^{1,2}$
}

It is crucial for the physical realization of quantum information networks to first establish entanglement among multiple space-separated quantum memories and then, at a user-controlled moment, to transfer the stored entanglement to quantum channels for distribution and conveyance of information. Here we present an experimental demonstration on generation, storage, and transfer of deterministic quantum entanglement among three spatially separated atomic ensembles. The off-line prepared multipartite entanglement of optical modes is mapped into three distant atomic ensembles to establish entanglement of atomic spin waves via electromagnetically induced transparency light-matter interaction. Then the stored atomic entanglement is transferred into a tripartite quadrature entangled state of light, which is space-separated and can be dynamically allocated to three quantum channels for conveying quantum information. The existence of entanglement among three released optical modes verifies that the system has the capacity to preserve multipartite entanglement. The presented protocol can be directly extended to larger quantum networks with more nodes.

\footnotetext{
${ }^{1}$ State Key Laboratory of Quantum Optics and Quantum Optics Devices, Institute of Opto-Electronics, Shanxi University, Taiyuan 030006, China.

${ }^{2}$ Collaborative Innovation Center of Extreme Optics, Shanxi University, Taiyuan 030006, China. Zhihui Yan and Liang Wu contributed equally to this work.

Correspondence and requests for materials should be addressed to X.J. (email: jiaxj@sxu.edu.cn)
} 
lying photons or bright optical beams are the best natural quantum channels, while usually matter systems are employed for memories at quantum nodes ${ }^{1,2}$. Single atoms $s^{3,4}$, atomic ensembles ${ }^{5-10}$, trapped ions ${ }^{11-13}$, optomechanics ${ }^{14-17}$, superconductors $^{18}$, solid-state systems ${ }^{19-22}$, and so on have been applied as quantum nodes. Especially, atomic ensembles are among the best candidates for quantum nodes to store and process quantum information due to the advantage of the collective enhancement of light-atom interaction ${ }^{5-10}$.

The entanglement of discrete quantum variables between two atomic ensembles has been experimentally achieved by means of Raman scattering approach ${ }^{23,24}$ or transferring quantum states of entangled photons into two atomic systems ${ }^{25-27}$. In 2010 , Kimble's group demonstrated measurement-induced entanglement stored in four atomic memories and coherent transfer of the atomic entanglement to four photonic channels ${ }^{28}$. For the first time, their experiment proved that a multipartite entangled $\mathrm{W}$ state of atomic ensembles can be transferred into a photonic mode $\mathrm{W}$ state with the heralded entanglement and thus showed an advance in the distribution of multipartite entanglement across quantum networks. Besides above-mentioned schemes based on applying discrete quantum variables of single photons and atoms, continuous-variable $(\mathrm{CV})$ regime provides another avenue toward the realization of quantum information tasks. To develop CV quantum information networks, CV entanglement between two macroscopic objects, i.e., atomic ensembles, has been investigated $^{29}, 30$. CV entanglement of spin wave variances between two atomic ensembles has been experimentally realized via quantum non-demolition measurement ${ }^{29}$ and dissipation mechanism of atomic systems ${ }^{30}$, respectively. For implementing quantum computation ${ }^{31}$ and quantum communication ${ }^{32}$, entanglement has to be stored in atomic memories and then to be controllably released on demand. Quantum memories for squeezing and entanglement of light have been theoretically investigated $^{33}, 34$, and the storage of CV entanglement between two atomic ensembles has been experimentally completed ${ }^{35}$. So far, all experiments on generation and storage of CV entanglement of atomic systems are concentrated between two ensembles $^{29,30,35}$. In ref. ${ }^{35}$, a displaced entangled state of two sideband modes of an optical beam is used for the initial quantum resource to create entanglement between two atomic ensembles. To extend this method to multipartite entanglement more sidebands with different frequency shifts have to be prepared and the number of entangled sideband modes must be strictly restricted by the bandwidth of optical parametric amplifier, which is the device for generating optical entangled state in their system. On the other hand, it is difficult to spatially separate these entangled optical submodes with small frequency intervals. Although a narrow band optical cavity can be utilized for separating these optical modes, entanglement among them will be significantly reduced ${ }^{36}$. These limitations make difficult to extend the experimental method of ref. ${ }^{35}$ to entangle more atomic ensembles. Up to now, it still remains a challenge to entangle more than two remote quantum memories in $\mathrm{CV}$ regime.

Here we present an experimental demonstration on deterministically establishing, storing, and releasing of CV entanglement among three atomic ensembles. At first, a tripartite optical entangled state is off-line prepared, and then the entanglement is

a

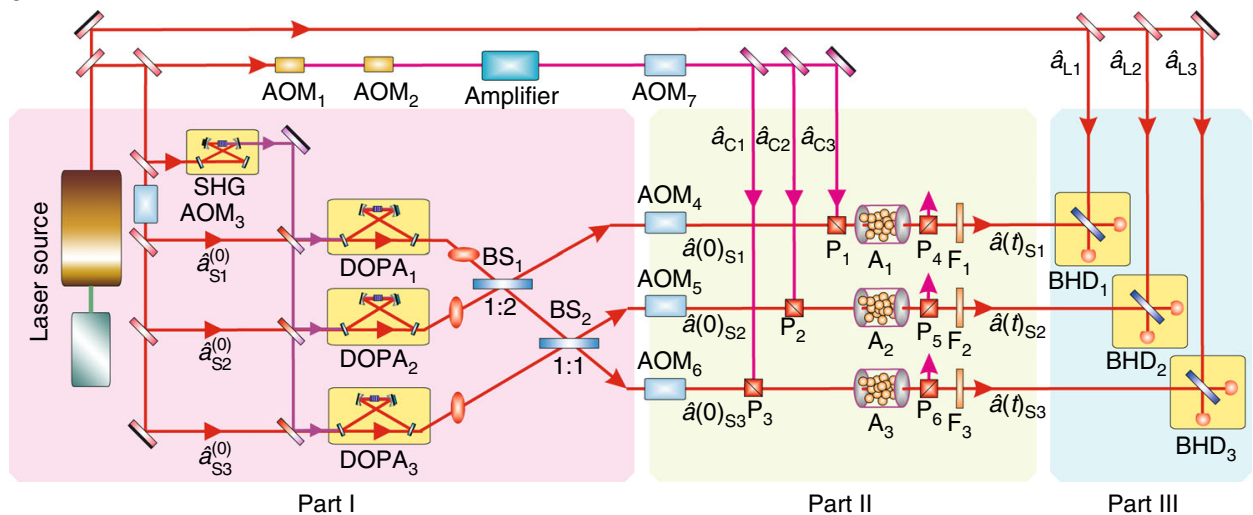

b

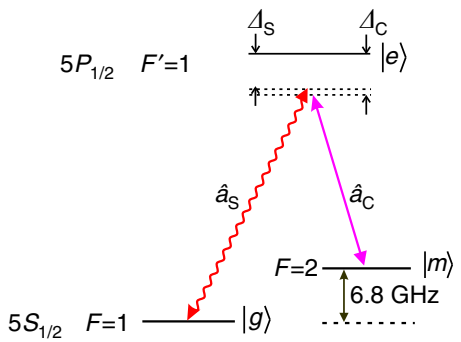

C

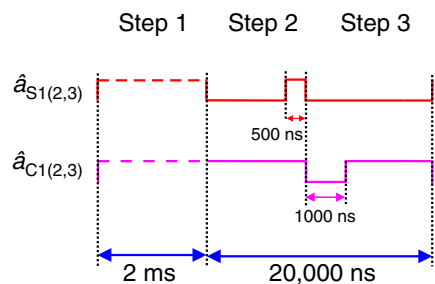

Fig. 1 Schematic diagram. a Experimental set-up. It includes three parts, Part I is the generation system of tripartite optical entanglement; Part II expresses the transportation of entanglement of optical modes to three distant atomic ensembles; Part III is the entanglement verification system. $\mathrm{A}_{1-3}$, atomic

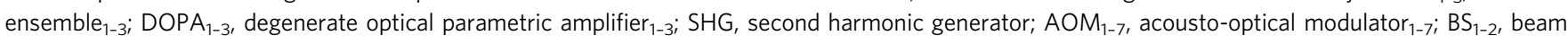

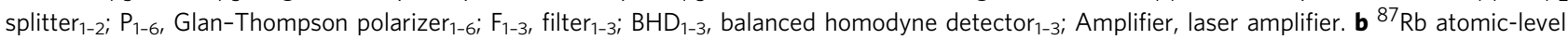
configuration and relevant transitions. $\left|5 S_{1 / 2}, F=1\right\rangle$ and $\left|5 S_{1 / 2}, F=2\right\rangle$ play the roles of ground state $|g\rangle$ and meta-state $|m\rangle$, respectively, and $\left|5 P_{1 / 2}, F^{\prime}=1\right\rangle$ is the excited state $|e\rangle$. Classical control optical beam $\hat{a}_{\mathrm{C}}$ (solid line) and quantum probe optical beam $\hat{a}_{\mathrm{S}}$ (wavy line) are shown. c Experimental time sequence for control optical beams $\hat{a}_{\mathrm{Cl}(2,3)}$ and signal optical beams $\hat{a}_{\mathrm{S} 1(2,3)}$ 
transferred into three atomic ensembles located $2.6 \mathrm{~m}$ apart from each other via electromagnetically induced transparency (EIT) interaction. After a given storage time, the preserved atomic entanglement is controllably released into three separated quantum channels consisting of three entangled optical submodes. The dependence of entanglement among three released optical submodes on systematic parameters is theoretically deduced and multipartite entanglement transfer as well as storage are experimentally proved. Since the tripartite optical entangled state is generated by linearly optical transformation of three squeezed states of light, its three submodes are naturally space separated ${ }^{37}$, 38 . The presented scheme can be directly extended to generate optical entangled states with more submodes if more squeezed states of light are available. In this way, entanglement of more atomic ensembles can be established.

\section{Results}

Experimental set-up. Figure 1a describes the experimental set-up for generation, storage, and transfer of tripartite entanglement. Three space separated submodes $\hat{a}(0)_{\mathrm{S} 1}, \hat{a}(0)_{\mathrm{S} 2}$, and $\hat{a}(0)_{\mathrm{S} 3}$ of an optical entangled state off-line prepared in Part I interact, respectively, with three atomic memories $A_{1}, A_{2}$, and $A_{3}$ located at three distant nodes to generate and store entanglement of spin waves among three atomic ensembles. Then the preserved entanglement is transferred back into an optical entangled state with three submodes $\hat{a}(t)_{\mathrm{S} 1}, \hat{a}(t)_{\mathrm{S} 2}$, and $\hat{a}(t)_{\mathrm{S} 3}$ after a storage time $t$ (Part II). At last, entanglement among three released optical submodes is measured by three balanced homodyne detectors $\mathrm{BHD}_{1-3}$ (Part III).

Three narrow band entangled optical beams tuned to the $\left|5 S_{1 / 2}, F=1\right\rangle \leftrightarrow\left|5 P_{1 / 2}, F^{\prime}=1\right\rangle$ transition of rubidium around $795 \mathrm{~nm}$ are obtained via linearly optical transformation of three squeezed states of light, which are generated by three degenerate optical parametric amplifiers $\left(\mathrm{DOPA}_{1-3}\right)$. DOPA 1 and $\mathrm{DOPA}_{2(3)}$ operating in parametric amplification and deamplification produce phase and amplitude quadrature squeezed states $\hat{a}_{\mathrm{S} 1}$ and $\hat{a}_{\mathrm{S} 2(3)}$, respectively ${ }^{39,40}$. The squeezing parameters $(r)$ for the three squeezed states are assumed to be identical for simplicity. In fact, three DOPAs used in our experiment have totally identical configuration and thus their squeezing parameters are almost the same. The quadrature amplitudes $\hat{X}_{\mathrm{S} j}=\left(\hat{a}_{\mathrm{Sj}}+\hat{a}_{\mathrm{Sj}}^{+}\right) / \sqrt{2}$ and phases $\hat{P}_{\mathrm{S} j}=\left(\hat{a}_{\mathrm{S} j}-\hat{a}_{\mathrm{Sj}}^{+}\right) / \sqrt{2} i(j=1,2,3)$ of output optical beams from three DOPAs are expressed as $\hat{X}_{\mathrm{S} 1}=\mathrm{e}^{r} \hat{X}_{\mathrm{S} 1}^{(0)}, \hat{P}_{\mathrm{S} 1}=\mathrm{e}^{-r} \hat{P}_{\mathrm{S} 1}^{(0)}$, $\hat{X}_{\mathrm{S} 2(3)}=\mathrm{e}^{-r} \hat{P}_{\mathrm{S} 1}^{(0)}$, and $\hat{P}_{\mathrm{S} 2(3)}=\mathrm{e}^{r} \hat{P}_{\mathrm{S} 2(3)}^{(0)}{ }^{40}$, where $\hat{X}(\hat{P})_{\mathrm{S} 1}^{(0)}, \hat{X}(\hat{P})_{\mathrm{S} 2}^{(0)}$, and $\hat{X}(\hat{P})_{\mathrm{S} 3}^{(0)}$ are amplitude (phase) quadratures of input optical beams $\hat{a}_{\mathrm{S} 1}^{(0)}, \hat{a}_{\mathrm{S} 2}^{(0)}$, and $\hat{a}_{\mathrm{S} 3}^{(0)}$ for DOPAs, respectively. By interfering three squeezed states of light on $\mathrm{BS}_{1}$ and $\mathrm{BS}_{2}$, we obtain a tripartite optical entangled state with quantum correlations of both amplitude quadratures $\hat{X}(0)_{\mathrm{Lj}}=\left(\hat{a}(0)_{\mathrm{S} j}+\hat{a}(0)_{\mathrm{Sj}}^{+}\right) / \sqrt{2}$ and phase quadratures $\hat{P}(0)_{\mathrm{Lj}}=\left(\hat{a}(0)_{\mathrm{S} j}-\hat{a}(0)_{\mathrm{S} j}^{+}\right) / \sqrt{2} i^{40}$. Then the three entangled optical beams are chopped into $500 \mathrm{~ns}$ pulses $\hat{a}(0)_{\mathrm{S} 1}, \hat{a}(0)_{\mathrm{S} 2}$, and $\hat{a}(0)_{\mathrm{S} 3}$, with three acoustical-optical modulators $\mathrm{AOM}_{4-6}$, which are used for the input signals of three atomic ensembles, i.e., ${ }^{87} \mathrm{Rb}$ vapor cells $A_{1}, A_{2}$, and $A_{3}$.

Quantum state transfer. The physical mechanism of light-matter interaction used for the experiment is EIT, which is a transparency phenomenon induced by optical field in an opaque medium by means of quantum interference ${ }^{41}$. M. Fleischhauer and M.D. Lukin have theoretically demonstrated that when quantum fields propagate in EIT media, there are form-stable quantum excitations associated with such propagation, named dark-state polaritons, and in this process the quantum state of

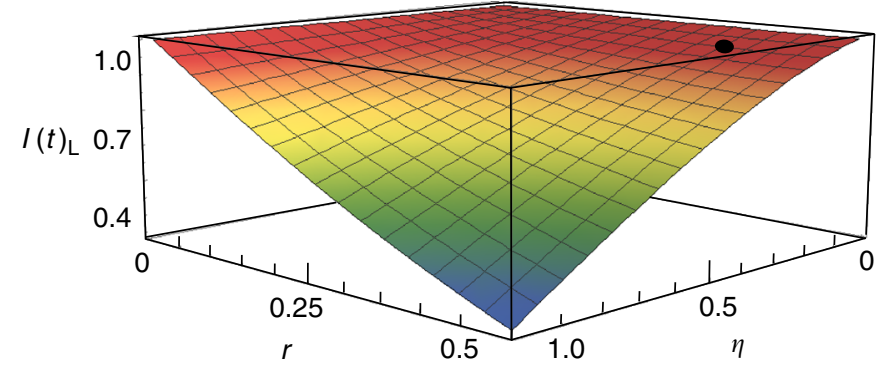

Fig. 2 The dependence of combinations of normalized quantum correlation variances among three released submodes after a storage time of $1000 \mathrm{~ns}$ on the squeezing parameter $r$ of three DOPAs and the total mapping efficiency $\eta$, where the gains $g_{\mathrm{L} 1-\mathrm{L} 3}^{\prime}$ are taken as the optimal gain $g_{\mathrm{L} 1-\mathrm{L} 3}^{\prime \text { opt }}$. The dot corresponds the experimental result of $I(t)_{\mathrm{L}}=0.96 \pm 0.01$, where the squeezing parameter $r$ is 0.38 and total mapping efficiency $\eta$ is about $16 \%$

light can be ideally transferred to collective atomic excitations and vice versa. Therefore, EIT effects can be applied to generate nonclassical states of atomic ensembles, to store optical quantum states, and reversibly to release stored quantum states into optical channels, respectively ${ }^{42-45}$. An atomic ensemble is represented by total angular momentum operator of collective atomic spins $\hat{J}=\sum_{i}|g\rangle\langle m|$, and $y, z$-components of the collective atomic angular momentum play the role of canonical variables, i.e., $\hat{X}_{\mathrm{A}}=\left(\hat{J}+\hat{J}^{+}\right) / \sqrt{2}=\hat{J}_{y} / \sqrt{\left\langle\hat{J}_{x}\right\rangle}$,

$\hat{P}_{\mathrm{A}}=\left(\hat{J}-\hat{J}^{+}\right) / \sqrt{2} i=\hat{J}_{z} / \sqrt{\left\langle\hat{J}_{x}\right\rangle^{35}}$. When control optical beams are adiabatically switched off, the mapping relations of amplitude (phase) quadratures from input optical submodes $\hat{X}(\hat{P})(0)_{\mathrm{L} j}$ to atomic spin waves $\hat{X}(\hat{P})(t)_{\mathrm{A} j}$ after a storage time $t$ are expressed by refs. ${ }^{46,47}$ :

$$
\begin{aligned}
\hat{X}(t)_{\mathrm{A} j} & =\sqrt{\eta_{\mathrm{M}}} \hat{X}(0)_{\mathrm{L} j}+\sqrt{1-\eta_{\mathrm{M}}} \hat{X}_{\mathrm{A} j}^{\mathrm{vac}}, \\
\hat{P}(t)_{\mathrm{A} j} & =\sqrt{\eta_{\mathrm{M}}} \hat{P}(0)_{\mathrm{L} j}+\sqrt{1-\eta_{\mathrm{M}}} \hat{P}_{\mathrm{Aj}}^{\mathrm{vac}},
\end{aligned}
$$

where the mapping efficiency from input optical submodes to atomic spin waves is $\eta_{\mathrm{M}}=\eta_{\mathrm{T}} \eta_{\mathrm{W}} \mathrm{e}^{-t / \tau_{\mathrm{s}}}, \eta_{\mathrm{T}}$ is the optical transmission efficiency, $\eta_{\mathrm{W}}$ is the storage efficiency of light in atomic ensemble, and $\tau_{\mathrm{s}}$ is the storage lifetime limited by atomic decoherence. The vacuum quadrature noises of atomic ensembles $\hat{X}(\hat{P})_{\mathrm{Aj}}^{\mathrm{vac}}$ are introduced by limited mapping efficiency $\eta_{\mathrm{M}}$. Since canonical quadrature operators of atomic spin waves obey the same commutation relation with that of Gaussian optical states, i.e., $\left[\hat{X}_{\mathrm{A}}, \hat{P}_{\mathrm{A}}\right]=i$, using similar procedure of deducing full tripartite inseparability criteria provided by Loock et al. ${ }^{48}$, we can obtain a set of analogous criterion inequalities for atomic spin waves (see also the "Methods" section).

The stored atomic entanglement can be transferred to tripartite entanglement among three output optical submodes $\hat{a}(t)_{\mathrm{S} 1}$, $\hat{a}(t)_{\mathrm{S} 2}$, and $\hat{a}(t)_{\mathrm{S} 3}$ after a storage time $t$ by turning on control optical beams. The quadrature amplitudes and phases of released submodes, $\hat{X}(t)_{\mathrm{Lj}}=\left(\hat{a}(t)_{\mathrm{S} j}+\hat{a}(t)_{\mathrm{Sj}}^{+}\right) / \sqrt{2}$ and $\hat{P}(t)_{\mathrm{L} j}=$ $\left(\hat{a}(t)_{S j}-\hat{a}(t)_{S j}^{+}\right) / \sqrt{2} i$ in terms of quadratures for atomic spin waves $\hat{X}(\hat{P})(t)_{\mathrm{Aj}}$ are expressed by refs. ${ }^{46,47}$ :

$$
\begin{gathered}
\hat{X}(t)_{\mathrm{L} j}=-\sqrt{\eta_{\mathrm{M}}^{\prime}} \hat{X}(t)_{\mathrm{A} j}+\sqrt{1-\eta_{\mathrm{M}}^{\prime}} \hat{X}_{\mathrm{L} j}^{\mathrm{vac}}, \\
\hat{P}(t)_{\mathrm{L} j}=-\sqrt{\eta_{\mathrm{M}}^{\prime}} \hat{P}(t)_{\mathrm{A} j}+\sqrt{1-\eta_{\mathrm{M}}^{\prime}} \hat{P}_{\mathrm{L} j}^{\mathrm{vac}},
\end{gathered}
$$




\section{Table 1 The values of normalized correlation variances for different combinations}

\begin{tabular}{llll} 
Correlation variances for different combinations & $\begin{array}{l}\text { Values for input submodes } \\
(\mathbf{d B})\end{array}$ & $\begin{array}{l}\text { Values for atomic spin } \\
\text { waves (dB) }\end{array}$ & $\begin{array}{l}\text { Values for released submodes } \\
\text { (dB) }\end{array}$ \\
\hline$\left\langle\delta^{2}\left(\hat{X}_{2}-\hat{X}_{3}\right)\right\rangle$ & $-3.30 \pm 0.05$ & $-0.56 \pm 0.03$ & $-0.37 \pm 0.03$ \\
$\left\langle\delta^{2}\left(g_{1} \hat{P}_{1}+\hat{P}_{2}+\hat{P}_{3}\right)\right\rangle$ & $-2.93 \pm 0.05$ & $-0.15 \pm 0.02$ & $-0.10 \pm 0.02$ \\
$\left\langle\delta^{2}\left(\hat{X}_{1}-\hat{X}_{3}\right)\right\rangle$ & $-3.25 \pm 0.05$ & $-0.53 \pm 0.03$ & $-0.35 \pm 0.03$ \\
$\left\langle\delta^{2}\left(\hat{P}_{1}+g_{2} \hat{P}_{2}+\hat{P}_{3}\right)\right\rangle$ & $-2.91 \pm 0.05$ & $-0.15 \pm 0.02$ & $-0.10 \pm 0.02$ \\
$\left\langle\delta^{2}\left(\hat{X}_{1}-\hat{X}_{2}\right)\right\rangle$ & $-3.25 \pm 0.05$ & $-0.52 \pm 0.03$ & $-0.34 \pm 0.03$ \\
$\left\langle\delta^{2}\left(\hat{P}_{1}+\hat{P}_{2}+g_{3} \hat{P}_{3}\right)\right\rangle$ & $-2.90 \pm 0.05$ & $-0.14 \pm 0.02$ & $-0.09 \pm 0.02$ \\
\hline
\end{tabular}

where the mapping efficiency from atomic spin waves to optical submodes $\eta_{\mathrm{M}}^{\prime}$ is the retrieval efficiency from atomic ensembles to light. The vacuum quadrature noises of optical submodes $\hat{X}(\hat{P})_{\mathrm{L} j}^{\mathrm{vac}}$ are introduced by the read process.

The full tripartite inseparability criteria for released optical modes are given by ref. $^{48}$ :

$$
\begin{aligned}
I(t)_{\mathrm{L} 1}= & \left\langle\delta^{2}\left(\hat{X}(t)_{\mathrm{L} 2}-\hat{X}(t)_{\mathrm{L} 3}\right)\right\rangle / 2+\left\langle\delta ^ { 2 } \left( g_{\mathrm{L} 1}^{\prime} \hat{P}(t)_{\mathrm{L} 1}\right.\right. \\
& \left.\left.+\hat{P}(t)_{\mathrm{L} 2}+\hat{P}(t)_{\mathrm{L} 3}\right)\right\rangle / 2 \geq 1, \\
I(t)_{\mathrm{L} 2}= & \left\langle\delta^{2}\left(\hat{X}(t)_{\mathrm{L} 1}-\hat{X}(t)_{\mathrm{L} 3}\right)\right\rangle / 2+\left\langle\delta ^ { 2 } \left(\hat{P}(t)_{\mathrm{L} 1}\right.\right. \\
& \left.\left.+g_{\mathrm{L} 2}^{\prime} \hat{P}(t)_{\mathrm{L} 2}+\hat{P}(t)_{\mathrm{L} 3}\right)\right\rangle / 2 \geq 1, \\
I(t)_{\mathrm{L} 3}= & \left\langle\delta^{2}\left(\hat{X}(t)_{\mathrm{L} 1}-\hat{X}(t)_{\mathrm{L} 2}\right)\right\rangle / 2+\left\langle\delta ^ { 2 } \left(\hat{P}(t)_{\mathrm{L} 1}\right.\right. \\
& \left.\left.+\hat{P}(t)_{\mathrm{L} 2}+g_{\mathrm{L} 3}^{\prime} \hat{P}(t)_{\mathrm{L} 3}\right)\right\rangle / 2 \geq 1 .
\end{aligned}
$$

If any two in the three inequalities are simultaneously violated, the three submodes form a tripartite Greenberger-Horne-Zeilinger-like (GHZ-like) entangled state of light, where $g_{\mathrm{L} 1}^{\prime}, g_{\mathrm{L} 2}^{\prime}$, and $g_{\mathrm{L} 3}^{\prime}$ are the gain factors for optimizing the correlation variances for released submodes. From Eqs. (1) and (2), it can be seen that entanglement is limited by total mapping efficiencies $\eta\left(\eta=\eta_{\mathrm{M}} \eta_{\mathrm{M}}^{\prime}\right)$ as well as squeezing parameter $r$. When the squeezing parameters $r$ for three DOPAs and the total mapping efficiencies $\eta$ for three atomic memories are the same, the values of left sides of three inequalities (3) are identical, $I(t)_{\mathrm{L} 1}=I(t)_{\mathrm{L} 2}=I(t)_{\mathrm{L} 3}=I(t)_{\mathrm{L}}$. The smaller $I(t)_{\mathrm{L}}$ is, the higher the entanglement is. Figure 2 shows the dependence of correlation variance combinations for three released submodes on the squeezing parameter $r$ of initial squeezed states and the total mapping efficiency $\eta$ with the storage time of $1000 \mathrm{~ns}$. We can see that the combinations of correlation variance are reduced with the increase of squeezing parameter $r$ and total mapping efficiency $\eta$ (see also the "Methods" section).

When the control optical beams $\hat{a}_{\mathrm{C} 1}, \hat{a}_{\mathrm{C} 2}$, and $\hat{a}_{\mathrm{C} 3}$, which are tuned to $\left|5 S_{1 / 2}, F=2\right\rangle \leftrightarrow\left|5 P_{1 / 2}, F^{\prime}=1\right\rangle$ transition of rubidium, are adiabatically switched off, optical entanglement among three input submodes $\hat{a}(0)_{\mathrm{S} 1}, \hat{a}(0)_{\mathrm{S} 2}$, and $\hat{a}(0)_{\mathrm{S} 3}$ is transferred to atomic entanglement of spin waves according to Eq. (1) via EIT interaction. After a storage time of $1000 \mathrm{ns,} \mathrm{control} \mathrm{optical} \mathrm{beams}$ $\hat{a}_{\mathrm{C} 1}, \hat{a}_{\mathrm{C} 2}$, and $\hat{a}_{\mathrm{C} 3}$ are switched on again, three optical submodes $\hat{a}(t)_{\mathrm{S} 1}, \hat{a}(t)_{\mathrm{S} 2}$, and $\hat{a}(t)_{\mathrm{S} 3}$ are released. The combinations of correlation variances in inequalities (3) are measured with three time domain $\mathrm{BHD}_{1-3}$. The intensive coherent light $\hat{a}_{\mathrm{L} 1}, \hat{a}_{\mathrm{L} 2}$, and $\hat{a}_{\mathrm{L} 3}$ are utilized as local oscillators of $\mathrm{BHD}_{1-3}$. The control and signal optical beams with orthogonally linear polarizations are combined on Glan-Thompson polarizers $\left(\mathrm{P}_{1-3}\right)$ before atomic cells, and control optical beams are filtered out from the signal optical beams by Glan-Thompson polarizers $\left(\mathrm{P}_{4-6}\right)$ and etalon filters $\left(\mathrm{F}_{1-3}\right)$. In storage and retrieval procedures 10,000 traces of $\mathrm{BHD}$ output signals with $20 \mathrm{G}$ samples/s are digitally filtered with a bandpass filter of $2.5 \mathrm{MHz}$ and averaged to obtain the optimal entangled degree. In this case, the low-frequency sideband noises resulting from pumping laser of DOPAs and atomic ensembles, as well as high frequency noises coming from parametric conversion in DOPAs and EIT process in atomic ensembles have been filtered out. When both control and signal optical beams are blocked and only the local oscillators are remained, outputs of BHDs stand for the corresponding vacuum noise level ${ }^{49}$.

Experimental results. The normalized correlation variances for different combinations of quadrature components are given in Table. 1, where gain factors $\left(g_{1}, g_{2}\right.$, and $\left.g_{3}\right)$ are chosen as the optimal gains for minimizing the corresponding correlation variances. The correlation variances of input and released submodes are directly measured with three sets of BHDs. The normalized correlation variances among three atomic ensembles are inferred from Eq. (2), where the mapping efficiency $\eta_{\mathrm{M}}^{\prime}$ is about $68 \%$ for our experimental system. The measured normalized quantum correlation variances are shown in Fig. 3. The squeezing parameter $r$ is 0.38 and total mapping efficiency $\eta$ is about $16 \%$. The combination of correlation variances for three released submodes is $I(t)_{\mathrm{L}}=0.96 \pm 0.01$, which is less than 1 , thus according to criterion inequalities (3) the entanglement among released submodes is verified. The value is in agreement with the theoretically calculated result, which is marked with a black dot in Fig. 2. Because of the limitation of total mapping efficiency, the correlation variances of released entangled state are much higher than that of input state. However, correlation variances below the corresponding vacuum noise level in Fig. 3 and the violation of criterion inequalities (3) certainly prove the existence of tripartite entanglement among three optical submodes released from atomic spin waves of three atomic ensembles. Thus the tripartite GHZ-like entanglement among three atomic ensembles is experimentally demonstrated.

\section{Discussion}

In summary, deterministic quantum entanglement among three spatially separated quantum nodes is experimentally generated, stored, and transferred. Within storage lifetime, the multipartite entanglement is preserved in three space separated atomic ensembles, and then at a desirable time the stored atomic entanglement can be controllably converted into three optical submodes to be quantum channels. Our work shows that multipartite CV entanglement can be established among remote macroscopic objects by transferring off-line prepared entanglement of optical beams into atomic ensembles via EIT interaction. Since unconditional CV entanglement among multipartite optical modes has been experimentally accomplished $37,50,51$, mature quantum optical technology can be used for realizing entanglement of more nodes in a quantum networks. The obtained 

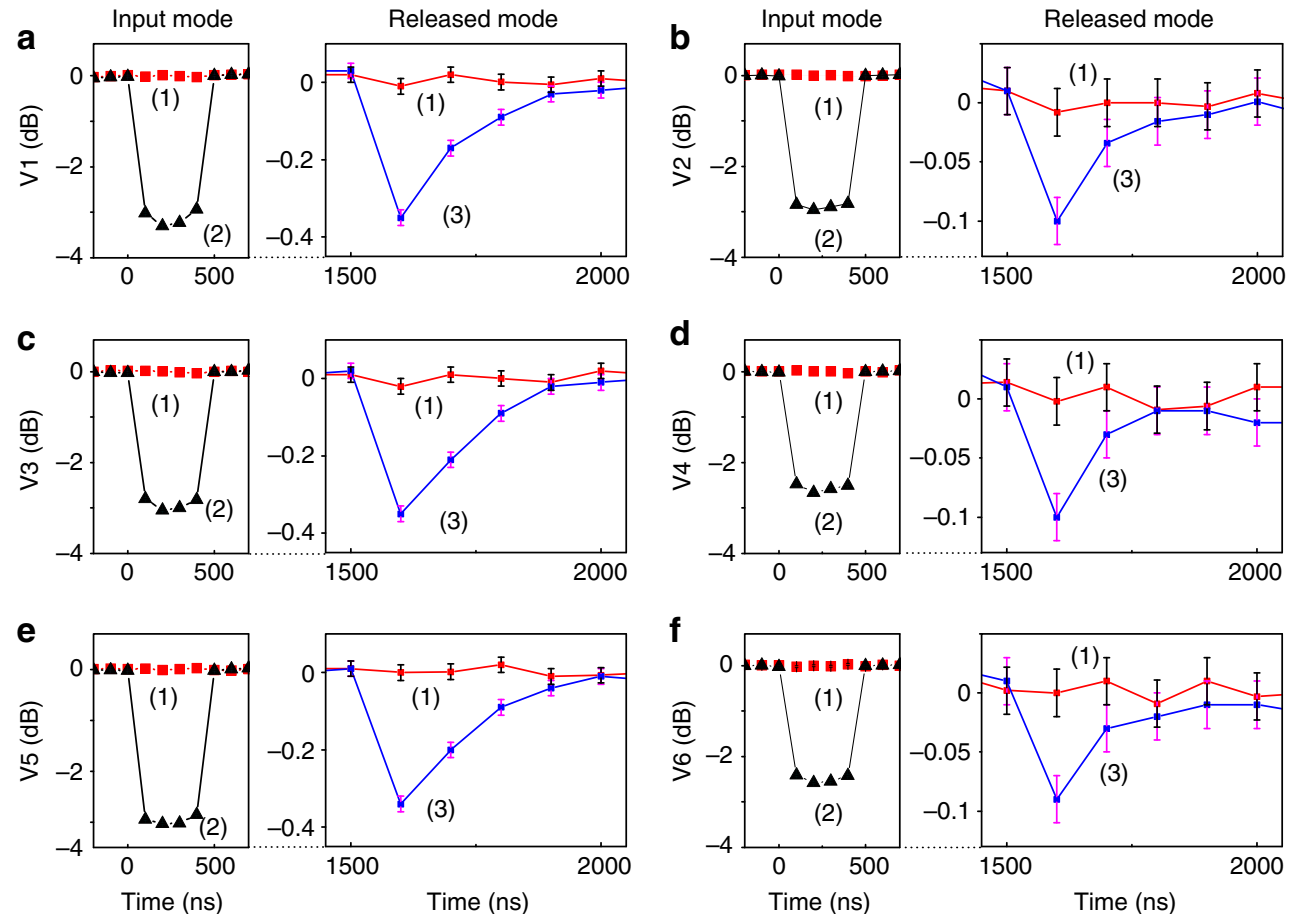

Fig. 3 Measured normalized correlation variances of input and released optical submodes. Trace (1) is the vacuum noise level. Trace (2) is the correlation variances of the original input optical submodes. a $\mathrm{V} 1\left(\left\langle\delta^{2}\left(\hat{X}(0)_{\mathrm{L} 2}-\hat{X}(0)_{\mathrm{L} 3}\right)\right\rangle\right), \mathbf{b} \mathrm{V} 2\left(\left\langle\delta^{2}\left(g_{\mathrm{L} 1}^{\text {opt }} \hat{P}(0)_{\mathrm{L} 1}+\hat{P}(0)_{\mathrm{L} 2}+\hat{P}(0)_{\mathrm{L} 3}\right)\right\rangle\right), \mathbf{c} \vee 3\left(\left\langle\delta^{2}\left(\hat{X}(0)_{\mathrm{L} 1}-\hat{X}(0)_{\mathrm{L} 3}\right)\right\rangle\right), \mathbf{d}$ V4 $\left(\left\langle\delta^{2}\left(\hat{P}(0)_{\mathrm{L} 1}+g_{\mathrm{L} 2}^{\text {opt }} \hat{P}(0)_{\mathrm{L} 2}+\hat{P}(0)_{\mathrm{L} 3}\right)\right\rangle\right)$, e $\mathrm{V} 5\left(\left\langle\delta^{2}\left(\hat{X}(0)_{\mathrm{L} 1}-\hat{X}(0)_{\mathrm{L} 2}\right)\right\rangle\right)$, $\mathbf{f} \mathrm{V} 6\left(\left\langle\delta^{2}\left(\hat{P}(0)_{\mathrm{L} 1}+\hat{P}(0)_{\mathrm{L} 2}+g_{\mathrm{L} 3}^{\text {opt }} \hat{P}(0)_{\mathrm{L} 3}\right)\right\rangle\right)$. Trace $(3)$ is corresponding correlation variances of released optical submodes after a storage time of $1000 \mathrm{~ns}$. Error bars represent \pm 1 standard error and are obtained with the statistics of the measured correlation variances

entanglement among atomic ensembles depends on mapping efficiency and initial squeezing parameter. The higher the squeezing and the mapping efficiency are, the better the entanglement among atomic ensembles is. In the presented experimental system, the total mapping efficiency is mainly limited by the optical transmission loss and memory (storage and retrieval) efficiency. The transmission loss (about 18\%) comes from the optical losses of atomic cells, etalon filters, Glan-Thompson polarizers and other optical components, which can be further reduced if better optical elements with lower losses are available.

The increased excess noises in released submodes from memories originate from fluorescence and coherent emission as well as spurious fluctuations in signal channels induced by the control optical beam ${ }^{44}$. On the other hand, since both EIT and four-wave mixing (FWM) effects are simultaneously generated in an ensemble of hot atoms ${ }^{52}$, the control optical beam acts as a fardetuned field on the signal transition in the undesired FWM process and spontaneously generates an "idler" field, which also can form excess noises. These mechanisms resulting in excess noises always exist in EIT light-matter interaction for any input state (vacuum state, squeezed vacuum state, entangled state or others). Therefore, some schemes used for improving EIT memory efficiency of classical signals ${ }^{52-54}$, such as decreasing detuning of probe and control optical beams, increasing the power of control optical beam and enhancing the temperature of atomic vapor, will unavoidably introduce more excess noises into atomic media and reduce quantum correlations among atomic ensembles. However, by optimizing experimental parameters, excess noises can be minimized for a given EIT experimental system $^{43,44,52}$.
It has been demonstrated that mapping efficiency can be significantly improved by means of the technology of optical cavity enhancement without introducing excess noise ${ }^{55,56}$ and that the storage lifetime can be dramatically increased if thermal atomic ensembles are replaced by cold atoms confined in threedimensional optical lattice 55 . Generation systems of optical squeezed states with squeezing up to $15 \mathrm{~dB}$ are available today ${ }^{57}$, providing initial high quality quantum resources for establishing better multipartite entanglement among atomic memories. The presented scheme opens up a new possibility for constructing future quantum internet ${ }^{1}$ and implementing distributed quantum computation based on the use of deterministic CV entanglement resources of light and atomic memories ${ }^{58}$.

\section{Methods}

Tripartite entangled state of light. A Ti:sapphire laser (Coherent MBR-110) pumped by green laser (Yuguang DPSS FG-VIIIB) outputs 3 W laser at about 795 $\mathrm{nm}$, which is used for pumping light of second harmonic generator (SHG) and seed lights of DOPAs. The configuration of optical cavities for SHG and three DOPAs is identical bow tie-type ring cavity with a $1 \times 2 \times 10 \mathrm{~mm}$ periodically poled KTiOPO4 (PPKTP) crystal. Three DOPAs are pumped by the second harmonic fields at about $398 \mathrm{~nm}$ from SHG and the fundamental waves from SHG are utilized as three injected seed fields $\hat{a}_{\mathrm{S} 1}^{(0)}, \hat{a}_{\mathrm{S} 2}^{(0)}$, and $\hat{a}_{\mathrm{S} 3}^{(0)}$. DOPA 1 and $\mathrm{DOPA}_{2(3)}$ are operated at parametric amplification and deamplification to produce quadrature phase- and amplitude-squeezed state of light, respectively ${ }^{8}$. The three squeezed optical beams are interfered on two optical beam splitters. The quadrature phasesqueezed field from $\operatorname{DOPA}_{1}\left(\hat{a}_{\mathrm{S} 1}\right)$ and the quadrature amplitude-squeezed field from $\operatorname{DOPA}_{2}\left(\hat{a}_{\mathrm{S} 2}\right)$ are first interfered on a beam splitter $\left(\mathrm{BS}_{1}\right)$ with the ratio of R:T $=1: 2$ (R: reflectivity and $\mathrm{T}$ : transmissivity). Then, one of two output optical beams from $\mathrm{BS}_{1}$ and the quadrature amplitude-squeezed light from $\operatorname{DOPA}_{3}\left(\hat{a}_{\mathrm{S} 3}\right)$ are interfered on $\mathrm{BS}_{2}$ with the ratio of $\mathrm{R}: \mathrm{T}=1: 1$. The relative phase between the two input optical beams on $\mathrm{BS}_{1(2)}$ is kept at $2 k \pi$ ( $k$ is integer). Finally, three entangled optical beams are chopped into three optical pulses $\hat{a}(0)_{\mathrm{S} 1}, \hat{a}(0)_{\mathrm{S} 2}$, and $\hat{a}(0)_{\mathrm{S} 3}$ by three $\mathrm{AOM}_{4-6}$. The three optical pulses are, respectively, injected into three atomic ensembles to be the input optical submodes. The quadrature's amplitudes and 


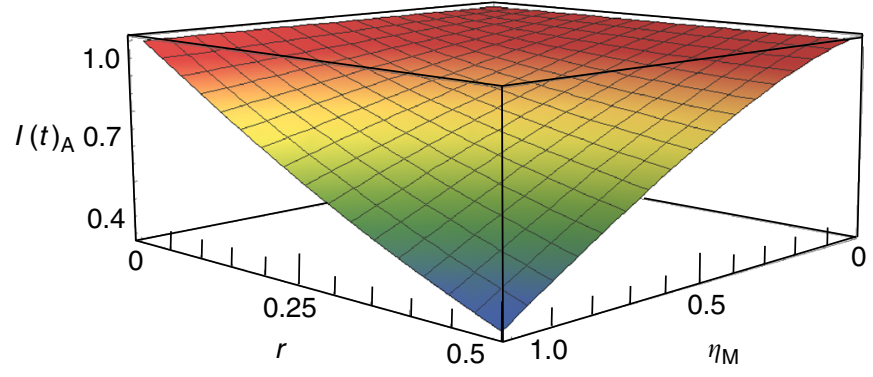

Fig. 4 The dependence of combination of normalized quantum correlation variances among three atomic ensembles after a storage time of $1000 \mathrm{~ns}$ on the squeezing parameter $r$ of three DOPAs and the mapping efficiency $\eta_{\mathrm{M}}$, where the gains are taken as the optimal gain $g_{\mathrm{A} 1-\mathrm{A} 3}^{\mathrm{opt}}$

phases of input optical submodes are expressed by ref. ${ }^{40}$ :

$$
\begin{aligned}
& \hat{X}(0)_{\mathrm{L} 1}=\sqrt{\frac{1}{3}} \mathrm{e}^{r} \hat{X}_{\mathrm{S} 1}^{(0)}+\sqrt{\frac{2}{3}} \mathrm{e}^{-r} \hat{X}_{\mathrm{S} 2}^{(0)}, \\
& \hat{P}(0)_{\mathrm{L} 1}=\sqrt{\frac{1}{3}} \mathrm{e}^{-r} \hat{P}_{\mathrm{S} 1}^{(0)}+\sqrt{\frac{2}{3}} \mathrm{e}^{r} \hat{P}_{\mathrm{S} 2}^{(0)}, \\
& \hat{X}(0)_{\mathrm{L} 2}=\sqrt{\frac{1}{3}} \mathrm{e}^{r} \hat{X}_{\mathrm{S} 1}^{(0)}-\sqrt{\frac{1}{6}} \mathrm{e}^{-r} \hat{X}_{\mathrm{S} 2}^{(0)}+\sqrt{\frac{1}{2}} \mathrm{e}^{-r} \hat{X}_{\mathrm{S} 3}^{(0)}, \\
& \hat{P}(0)_{\mathrm{L} 2}=\sqrt{\frac{1}{3}} \mathrm{e}^{-r} \hat{P}_{\mathrm{S} 1}^{(0)}-\sqrt{\frac{1}{6}} \mathrm{e}^{r} \hat{P}_{\mathrm{S} 2}^{(0)}+\sqrt{\frac{1}{2}} \mathrm{e}^{r} \hat{P}_{\mathrm{S} 3}^{(0)} \text {, } \\
& \hat{X}(0)_{\mathrm{L} 3}=\sqrt{\frac{1}{3}} \mathrm{e}^{r} \hat{X}_{\mathrm{S} 1}^{(0)}-\sqrt{\frac{1}{6}} \mathrm{e}^{-r} \hat{X}_{\mathrm{S} 2}^{(0)}-\sqrt{\frac{1}{2}} \mathrm{e}^{-r} \hat{X}_{\mathrm{S} 3}^{(0)}, \\
& \hat{P}(0)_{\mathrm{L} 3}=\sqrt{\frac{1}{3}} \mathrm{e}^{-r} \hat{P}_{\mathrm{S} 1}^{(0)}-\sqrt{\frac{1}{6}} \mathrm{e}^{r} \hat{P}_{\mathrm{S} 2}^{(0)}-\sqrt{\frac{1}{2}} \mathrm{e}^{r} \hat{P}_{\mathrm{S} 3}^{(0)},
\end{aligned}
$$

respectively.

The inequalities of full inseparability criteria for input tripartite entangled states of light are ref. ${ }^{48}$,

$$
\begin{aligned}
& I(0)_{\mathrm{L} 1}=\left\langle\delta^{2}\left(\hat{X}(0)_{\mathrm{L} 2}-\hat{X}(0)_{\mathrm{L} 3}\right)\right\rangle / 2+\left\langle\delta^{2}\left(g_{\mathrm{L} 1} \hat{P}(0)_{\mathrm{L} 1}+\hat{P}(0)_{\mathrm{L} 2}+\hat{P}(0)_{\mathrm{L} 3}\right)\right\rangle / 2 \geq 1, \\
& I(0)_{\mathrm{L} 2}=\left\langle\delta^{2}\left(\hat{X}(0)_{\mathrm{L} 1}-\hat{X}(0)_{\mathrm{L} 3}\right)\right\rangle / 2+\left\langle\delta^{2}\left(\hat{P}(0)_{\mathrm{L} 1}+g_{\mathrm{L} 2} \hat{P}(0)_{\mathrm{L} 2}+\hat{P}(0)_{\mathrm{L} 3}\right)\right\rangle / 2 \geq 1, \\
& I(0)_{\mathrm{L} 3}=\left\langle\delta^{2}\left(\hat{X}(0)_{\mathrm{L} 1}-\hat{X}(0)_{\mathrm{L} 2}\right)\right\rangle / 2+\left\langle\delta^{2}\left(\hat{P}(0)_{\mathrm{L} 1}+\hat{P}(0)_{\mathrm{L} 2}+g_{\mathrm{L} 3} \hat{P}(0)_{\mathrm{L} 3}\right)\right\rangle / 2 \geq 1 .
\end{aligned}
$$

If any two in the three inequalities are simultaneously violated, the three submodes form a tripartite GHZ-like entangled state, where $g_{\mathrm{L} 1}, g_{\mathrm{L} 2}$, and $g_{\mathrm{L} 3}$ are gain factors for minimizing correlation variances of the input tripartite entangled state of light.

When the squeezing parameter $r(r \geq 0)$ for three DOPAs is the same, the gain factors in inequalities (5) should be the same, i.e., $g_{\mathrm{L} 1}=g_{\mathrm{L} 2}=g_{\mathrm{L} 3}=g_{\mathrm{L}}$, and the values of left sides of three inequalities are identical, i.e., $I(0)_{\mathrm{L} 1}=I(0)_{\mathrm{L} 2}=I(0)_{\mathrm{L} 3}=$ $I(0)_{\text {L. }}$. Using Eq. (4), the combination of normalized quantum correlation variances for input optical beams is obtained:

$$
I(0)_{\mathrm{L}}=\frac{12 \mathrm{e}^{-2 r}+2\left(g_{\mathrm{L}}+2\right)^{2} \mathrm{e}^{-2 r}+4\left(g_{\mathrm{L}}-1\right)^{2} \mathrm{e}^{2 r}}{24} .
$$

Calculating the minimum value of Eq. (6), we get the optimal gain factor $g_{\mathrm{L}}^{\text {opt }}$

$$
g_{\mathrm{L}}^{\mathrm{opt}}=\frac{2 \mathrm{e}^{4 r}-2}{2 \mathrm{e}^{4 r}+1} \text {. }
$$

If squeezing parameter $r$ is larger than 0 , the combination of correlation variances will be less than 1 with the optimal gain factor, and the input optical submodes of atomic ensembles are in a tripartite GHZ-like entangled state.

Establishing tripartite atomic ensemble entanglement. In EIT memory medium, quantum state can be mapped from input optical submode $\hat{a}(0)_{\mathrm{S}}$ into atomic spin wave $\hat{J}$ and vice versa under the interaction with a strong control optical beam $\hat{a}_{C}{ }^{41,42}$. The control optical beam is treated as classical optical beam $A_{\mathrm{C}}$ because it is much more intensive than the signal optical modes. In EIT process, the effective interaction Hamiltonian $\hat{H}_{\mathrm{EIT}}$ between signal optical mode $\hat{a}(0)_{\mathrm{S}}$ and atomic spin wave $\hat{J}$ is given by refs. ${ }^{2,} 46$ :

$$
\hat{H}_{\mathrm{EIT}}=i \hbar \kappa A_{\mathrm{C}} \hat{a}(0)_{\mathrm{S}} \hat{J}^{+}-i \hbar \kappa A_{\mathrm{C}} \hat{a}(0)_{\mathrm{S}}^{+} \hat{J},
$$

which is similar to a beam splitter interaction, where $\kappa$ stands for the interaction constant between light and atoms.
By solving Heisenberg motion equations with the Hamiltonian $\hat{H}_{\text {EIT }}$ (Eq. 8), we obtain the expressions of quantum storage process and the mapping relations (Eq. 1) of amplitude and phase quadratures from input optical submodes $\hat{X}(\hat{P})(0)_{\mathrm{L} j}$ to atomic spin waves $\hat{X}(\hat{P})(t)_{\mathrm{Aj}}$ after a storage time $t$.

When the control optical beams are turned on, the input signal submodes are compressed in atomic ensembles due to the slow propagation under EIT interaction. On the moment of simultaneously shutting off three control optical beams, quantum entanglement among three pulse submodes will be mapped into atomic spin waves in the three ensembles, where the control optical beam plays the role of writing process. Using Eqs. (1) and (4), the amplitude (phase) quadratures of atomic spin waves $\hat{X}(\hat{P})(t)_{\mathrm{Aj}}$ after a storage time $t$ are obtained:

$$
\begin{array}{r}
\hat{X}(t)_{\mathrm{A} 1}=\sqrt{\frac{\eta_{\mathrm{M}}}{3}} \mathrm{e}^{r} \hat{X}_{\mathrm{S} 1}^{(0)}+\sqrt{\frac{2 \eta_{\mathrm{M}}}{3}} \mathrm{e}^{-r} \hat{X}_{\mathrm{S} 2}^{(0)}+\sqrt{1-\eta_{\mathrm{M}}} \hat{X}_{\mathrm{A} 1}^{\mathrm{vac}}, \\
\hat{P}(t)_{\mathrm{A} 1}=\sqrt{\frac{\eta_{\mathrm{M}}}{3}} \mathrm{e}^{-r} \hat{P}_{\mathrm{S} 1}^{(0)}+\sqrt{\frac{2 \eta_{\mathrm{M}}}{3}} \mathrm{e}^{r} \hat{P}_{\mathrm{S} 2}^{(0)}+\sqrt{1-\eta_{\mathrm{M}}} \hat{P}_{\mathrm{A} 1}^{\mathrm{vac}}, \\
\hat{X}(t)_{\mathrm{A} 2}=\sqrt{\frac{\eta_{\mathrm{M}}}{3}} \mathrm{e}^{r} \hat{X}_{\mathrm{S} 1}^{(0)}-\sqrt{\frac{\eta_{\mathrm{M}}}{6}} \mathrm{e}^{-r} \hat{X}_{\mathrm{S} 2}^{(0)}+\sqrt{\frac{\eta_{\mathrm{M}}}{2}} \mathrm{e}^{-r} \hat{X}_{\mathrm{S} 3}^{(0)}+\sqrt{1-\eta_{\mathrm{M}}} \hat{X}_{\mathrm{A} 2}^{\mathrm{vac}}, \\
\hat{P}(t)_{\mathrm{A} 2}=\sqrt{\frac{\eta_{\mathrm{M}}}{3}} \mathrm{e}^{-r} \hat{P}_{\mathrm{S} 1}^{(0)}-\sqrt{\frac{\eta_{\mathrm{M}}}{6}} \mathrm{e}^{r} \hat{P}_{\mathrm{S} 2}^{(0)}+\sqrt{\frac{\eta_{\mathrm{M}}}{2}} \mathrm{e}^{r} \hat{P}_{\mathrm{S} 3}^{(0)}+\sqrt{1-\eta_{\mathrm{M}}} \hat{P}_{\mathrm{A} 2}^{\mathrm{vac}}, \\
\hat{X}(t)_{\mathrm{A} 3}=\sqrt{\frac{\eta_{\mathrm{M}}}{3}} \mathrm{e}^{r} \hat{X}_{\mathrm{S} 1}^{(0)}-\sqrt{\frac{\eta_{\mathrm{M}}}{6}} \mathrm{e}^{-r} \hat{X}_{\mathrm{S} 2}^{(0)}-\sqrt{\frac{\eta_{\mathrm{M}}}{2}} \mathrm{e}^{-r} \hat{X}_{\mathrm{S} 3}^{(0)}+\sqrt{1-\eta_{\mathrm{M}}} \hat{X}_{\mathrm{A} 3}^{\mathrm{vac}}, \\
\hat{P}(t)_{\mathrm{A} 3}=\sqrt{\frac{\eta_{\mathrm{M}}}{3}} \mathrm{e}^{-r} \hat{P}_{\mathrm{S} 1}^{(0)}-\sqrt{\frac{\eta_{\mathrm{M}}}{6}} \mathrm{e}^{r} \hat{P}_{\mathrm{S} 2}^{(0)}-\sqrt{\frac{\eta_{\mathrm{M}}}{2}} \mathrm{e}^{r} \hat{P}_{\mathrm{S} 3}^{(0)}+\sqrt{1-\eta_{\mathrm{M}}} \hat{P}_{\mathrm{A} 3}^{\mathrm{vac}},
\end{array}
$$

respectively.

Since canonical quadrature operators of atomic spin waves obey the same commutation relation with that of Gaussian optical states, i.e., $\left[\hat{X}_{\mathrm{A}}, \hat{P}_{\mathrm{A}}\right]=i$, using similar procedure of deducing full tripartite inseparability criteria provided by Loock et al. ${ }^{48}$, we obtain a set of analogous criterion inequalities for atomic spin waves:

$$
\begin{aligned}
& I(t)_{\mathrm{A} 1}=\left\langle\delta^{2}\left(\hat{X}(t)_{\mathrm{A} 2}-\hat{X}(t)_{\mathrm{A} 3}\right)\right\rangle / 2+\left\langle\delta^{2}\left(g_{\mathrm{A} 1} \hat{P}(t)_{\mathrm{A} 1}+\hat{P}(t)_{\mathrm{A} 2}+\hat{P}(t)_{\mathrm{A} 3}\right)\right\rangle / 2 \geq 1, \\
& I(t)_{\mathrm{A} 2}=\left\langle\delta^{2}\left(\hat{X}(t)_{\mathrm{A} 1}-\hat{X}(t)_{\mathrm{A} 3}\right)\right\rangle / 2+\left\langle\delta^{2}\left(\hat{P}(t)_{\mathrm{A} 1}+g_{\mathrm{A} 2} \hat{P}(t)_{\mathrm{A} 2}+\hat{P}(t)_{\mathrm{A} 3}\right)\right\rangle / 2 \geq 1, \\
& I(t)_{\mathrm{A} 3}=\left\langle\delta^{2}\left(\hat{X}(t)_{\mathrm{A} 1}-\hat{X}(t)_{\mathrm{A} 2}\right)\right\rangle / 2+\left\langle\delta^{2}\left(\hat{P}(t)_{\mathrm{A} 1}+\hat{P}(t)_{\mathrm{A} 2}+g_{\mathrm{A} 3} \hat{P}(t)_{\mathrm{A} 3}\right)\right\rangle / 2 \geq 1 .
\end{aligned}
$$

When any two in three inequalities are violated, the three atomic ensembles are in an entangled state of GHZ-like type, where $g_{\mathrm{A} 1}, g_{\mathrm{A} 2}$, and $g_{\mathrm{A} 3}$ are gain factors for atomic ensembles.

If the mapping efficiencies $\eta_{\mathrm{M}}$ for three atomic memories are the same, the gain factors in inequalities (10) should be the same, i.e., $g_{\mathrm{A} 1}=g_{\mathrm{A} 2}=g_{\mathrm{A} 3}=g_{\mathrm{A}}$, and the values of three inequalities are also identical, i.e., $I(t)_{\mathrm{A} 1}=I(t)_{\mathrm{A} 2}=I(t)_{\mathrm{A} 3}=I(t)_{\mathrm{A}}$. According to Eq. (9), the combination of normalized quantum correlation variances for atomic spin waves after a storage time $t$ is obtained:

$$
I(t)_{\mathrm{A}}=\frac{12 \mathrm{e}^{-2 r}+2\left(g_{\mathrm{A}}+2\right)^{2} \mathrm{e}^{-2 r}+4\left(g_{\mathrm{A}}-1\right)^{2} \mathrm{e}^{2 r}}{24} \eta_{\mathrm{M}}+\left(1+\frac{1}{4} g_{\mathrm{A}}\right)\left(1-\eta_{\mathrm{M}}\right) .
$$

Similarly, by minimizing $I(t)_{\mathrm{A}}$ we get the optimal gain factor $g_{\mathrm{A}}^{\text {opt }}$ :

$$
g_{\mathrm{A}}^{\text {opt }}=\frac{2 \eta_{\mathrm{M}} \mathrm{e}^{4 r}-2 \eta_{\mathrm{M}}}{3 \mathrm{e}^{2 r}+\eta_{\mathrm{M}}-3 \eta_{\mathrm{M}} \mathrm{e}^{2 r}+2 \mathrm{e}^{4 r} \eta_{\mathrm{M}}} .
$$

The smaller $I(t)_{\mathrm{A}}$ is, the better the atomic entanglement is. Figure 4 shows the dependence of correlation variance combination for three atomic ensembles on the squeezing parameter $r$ of initial squeezed states and the mapping efficiency $\eta_{\mathrm{M}}$ with the storage time of $1000 \mathrm{~ns}$. We can see that the combination is reduced with the increase of squeezing parameter $r$ and mapping efficiency $\eta_{\mathrm{M}}$. In our experiment, the squeezing parameter $r$ and the mapping efficiency $\eta_{\mathrm{M}}$ are about 0.38 and $23 \%$, respectively.

Quantum state transfer of stored entangled state. The control optical beams of read process enable to map quantum state from the atomic spin waves to released optical submodes. Using Eqs. (2) and (9), the amplitude (phase) quadratures $\hat{X}(\hat{P})(t)_{\mathrm{L} j}$ of released optical submodes after a storage time $t$ are calculated. We 
have:

$$
\begin{array}{r}
\hat{X}(t)_{\mathrm{L} 1}=\sqrt{\frac{\eta}{3}} \mathrm{e}^{r} \hat{X}_{\mathrm{S} 1}^{(0)}+\sqrt{\frac{2 \eta}{3}} \mathrm{e}^{-r} \hat{X}_{\mathrm{S} 2}^{(0)}+\sqrt{1-\eta} \hat{X}_{\mathrm{L} 1}^{\mathrm{vac}}, \\
\hat{P}(t)_{\mathrm{L} 1}=\sqrt{\frac{\eta}{3}} \mathrm{e}^{-r} \hat{P}_{\mathrm{S} 1}^{(0)}+\sqrt{\frac{2 \eta}{3}} \mathrm{e}^{r} \hat{P}_{\mathrm{S} 2}^{(0)}+\sqrt{1-\eta} \hat{P}_{\mathrm{L} 1}^{\mathrm{vac}}, \\
\hat{X}(t)_{\mathrm{L} 2}=\sqrt{\frac{\eta}{3}} \mathrm{e}^{r} \hat{X}_{\mathrm{S} 1}^{(0)}-\sqrt{\frac{\eta}{6}} \mathrm{e}^{-r} \hat{X}_{\mathrm{S} 2}^{(0)}+\sqrt{\frac{\eta}{2}} \mathrm{e}^{-r} \hat{X}_{\mathrm{S} 3}^{(0)}+\sqrt{1-\eta} \hat{X}_{\mathrm{L} 2}^{\mathrm{vac}}, \\
\hat{P}(t)_{\mathrm{L} 2}=\sqrt{\frac{\eta}{3}} \mathrm{e}^{-r} \hat{P}_{\mathrm{S} 1}^{(0)}-\sqrt{\frac{\eta}{6}} \mathrm{e}^{r} \hat{P}_{\mathrm{S} 2}^{(0)}+\sqrt{\frac{\eta}{2}} \mathrm{e}^{r} \hat{P}_{\mathrm{S} 3}^{(0)}+\sqrt{1-\eta} \hat{P}_{\mathrm{L} 2}^{\mathrm{vac}}, \\
\hat{X}(t)_{\mathrm{L} 3}=\sqrt{\frac{\eta}{3}} \mathrm{e}^{r} \hat{X}_{\mathrm{S} 1}^{(0)}-\sqrt{\frac{\eta}{6}} \mathrm{e}^{-r} \hat{X}_{\mathrm{S} 2}^{(0)}-\sqrt{\frac{\eta}{2}} \mathrm{e}^{-r} \hat{X}_{\mathrm{S} 3}^{(0)}+\sqrt{1-\eta} \hat{X}_{\mathrm{L} 3}^{\mathrm{vac}}, \\
\hat{P}(t)_{\mathrm{L} 3}=\sqrt{\frac{\eta}{3}} \mathrm{e}^{-r} \hat{P}_{\mathrm{S} 1}^{(0)}-\sqrt{\frac{\eta}{6}} \mathrm{e}^{r} \hat{P}_{\mathrm{S} 2}^{(0)}-\sqrt{\frac{\eta}{2}} \mathrm{e}^{r} \hat{P}_{\mathrm{S} 3}^{(0)}+\sqrt{1-\eta} \hat{P}_{\mathrm{L} 3}^{\mathrm{vac}} .
\end{array}
$$

In this case of $g_{\mathrm{L} 1}^{\prime}=g_{\mathrm{L} 2}^{\prime}=g_{\mathrm{L} 3}^{\prime}=g_{\mathrm{L}}^{\prime}, I(t)_{\mathrm{L}}$ in inequalities (3) can be calculated from Eq. (13):

$$
I(t)_{\mathrm{L}}=\frac{12 \mathrm{e}^{-2 r}+2\left(g_{\mathrm{L}}^{\prime}+2\right)^{2} \mathrm{e}^{-2 r}+4\left(g_{\mathrm{L}}^{\prime}-1\right)^{2} \mathrm{e}^{2 r}}{24} \eta+\left(1+\frac{1}{4} g_{\mathrm{L}}^{\prime}\right)(1-\eta)
$$

The optimal gain factor $\left(g_{\mathrm{L}}^{\prime{ }^{\mathrm{opt}}}\right)$ for read out process equals to:

$$
g_{\mathrm{L}}^{\prime \mathrm{opt}^{2}}=\frac{2 \eta \mathrm{e}^{4 r}-2 \eta_{\mathrm{M}}^{\prime}}{3 \mathrm{e}^{2 r}+\eta-3 \eta \mathrm{e}^{2 r}+2 \mathrm{e}^{4 r} \eta}
$$

Figure 2 is obtained from Eq. (14), where the optimal gain in Eq. (15) is applied.

Atomic ensemble. The atomic energy levels of rubidium used for quantum memory medium is illustrated in Fig. $3 \mathrm{~b}$. The collective coherence of the ground state $\left|5 S_{1 / 2}, F=1\right\rangle$ and meta-state $\left|5 S_{1 / 2}, F=2\right\rangle$ is used to store nonclassical state of light. For balancing the storage efficiency and excess noises from atoms, the frequencies of both signal and control optical beams are detuned by $\Delta_{s}=700 \mathrm{MHz}$ (the detuning of signal optical beam from the transition between energy levels $g$ and e) and $\Delta_{c}=700.5 \mathrm{MHz}$ (the detuning of control optical beam from the transition between energy levels $\mathrm{m}$ and $\mathrm{e}$ ), respectively. The detuning is realized by two sets of double-pass $1.7 \mathrm{GHz}$ acousto-optical modulators $\left(\mathrm{AOM}_{1-2}\right)$. Three rubidium vapor cells of $7.5 \mathrm{~cm}$-long with 10 torr of neon buffer gas in a $\mu$ metal magnetic shielding are used as atomic media, where the neon buffer gas prevents thermal diffusion to increase the atomic coherence. The three rubidium atomic cells are heated to around $65^{\circ} \mathrm{C}$ in our experiment.

Experimental time sequence. In the beginning of each period, the laser is turned on for $2 \mathrm{~ms}$ by $\mathrm{AOM}_{3}$ and split into three optical beams $\hat{a}_{\mathrm{S} 1}^{(0)}, \hat{a}_{\mathrm{S} 2}^{(0)}$, and $\hat{a}_{\mathrm{S} 3}^{(0)}$, which are used as input signals of DOPAs, respectively, for persistently locking the length of optical cavities. Then the input signals of DOPAs are turned off for 20,000 ns by $\mathrm{AOM}_{3}$ to generate three squeezed vacuum states of light. Within the locking period the three entangled optical submodes are chopped into $500 \mathrm{~ns}$ pulses with $\mathrm{AOM}_{4-6}$, which are used for input submodes $\hat{a}(0)_{\mathrm{S} 1}, \hat{a}(0)_{\mathrm{S} 2}$, and $\hat{a}(0)_{\mathrm{S} 3}$ of three atomic ensembles. Once the signal pulses enter into the atomic cell, the control optical beams $\hat{a}_{C 1}, \hat{a}_{C_{2}}$, and $\hat{a}_{C 3}$ are switched off by $\mathrm{AOM}_{7}$ to complete the quantum storage. At a user-controlled moment (1000 ns for our experiment) within storage lifetime, the control optical beams $\hat{a}_{\mathrm{C} 1}, \hat{a}_{\mathrm{C} 2}$, and $\hat{a}_{\mathrm{C} 3}$ are turned on again by $\mathrm{AOM}_{7}$ at to obtain three released optical pulses $\hat{a}(t)_{\mathrm{S} 1}, \hat{a}(t)_{\mathrm{S} 2}$, and $\hat{a}(t)_{\mathrm{S} 3}$.

Data availability. The data that support the findings of this study are available from the corresponding author on request.

Received: 8 March 2017 Accepted: 28 July 2017

Published online: 28 September 2017

\section{References}

1. Kimble, H. J. The quantum internet. Nature 453, 1023-1030 (2008).

2. Hammerer, K., Sorensen, A. S. \& Polzik, E. S. Quantum interface between light and atomic ensembles. Rev. Mod. Phys. 82, 1041-1093 (2008).

3. Specht, H. et al. A single-atom quantum memory. Nature 473, 190-193 (2011).

4. Facon, A. et al. A sensitive electrometer based on a Rydberg atom in a Schrodinger-cat state. Nature 535, 262-265 (2016).

5. Simon, J., Tanji, H., Ghosh, S. \& Vuletic, V. Single-photon bus connecting spin-wave quantum memories. Nat. Phys. 3, 765-769 (2007).
6. Marino, A. M., Pooser, R. C., Boyer, V. \& Lett, P. D. Tunable delay of Einstein-Podolsky-Rosen entanglement. Nature 457, 859-862 (2009).

7. Hosseini, M., Sparkes, B. M., Campbell, G., Lam, P. K. \& Buchler, B. C. High efficiency coherent optical memory with warm rubidium vapour. Nat. Commun. 2, 174 (2011).

8. Xu, Z. X. et al. Long lifetime and high-fidelity quantum of photonic polarization qubit by lifting Zeeman degeneracy. Phys. Rev. Lett. 111, 240503 (2013).

9. Chen, Y. H. et al. Coherent optical memory with high storage efficiency and large fractional delay. Phys. Rev. Lett. 110, 083601 (2013).

10. Parigi, V. et al. Storage and retrieval of vector beams of light in a multipledegree-of-freedom quantum memory. Nat. Commun. 6, 7706 (2015).

11. Langer, C. et al. Long-lived qubit memory using atomic ions. Phys. Rev. Lett. 95 060502 (2005).

12. Stute, A. et al. Tunable ion-photon entanglement in an optical cavity. Nature 485, 482-485 (2012).

13. Hucul, D. et al. Modular entanglement of atomic qubits using photons and phonons. Nat. Phys. 11, 37-42 (2014).

14. Fiore, V. et al. Storing optical information as a mechanical excitation in a silica optomechanical resonator. Phys. Rev. Lett. 107, 133601 (2011).

15. Lee, H. et al. Spiral resonators for on-chip laser frequency stabilization. Nat. Commun. 4, 2468 (2013).

16. Riedinger, R. et al. Nonclassical correlations between single photons and phonons from a mechanical oscillator. Nature 530, 313-316 (2016).

17. Kiesewetter, S., Teh, R. Y., Reid, M. D. \& Drummond, P. D. Pulsed Entanglement of Two Optomechanical Oscillators and Furry's Hypothesis. Phys. Rev. Lett. 119, 023601 (2017).

18. Flurin, E., Roch, N., Pillet, J., Mallet, F. \& Huard, B. Superconducting quantum node for entanglement and storage of microwave radiation. Phys. Rev. Lett. 114, 090503 (2015).

19. Clausen, C. et al. Quantum storage of photonic entanglement in a crystal. Nature 469, 508-511 (2011).

20. Saglamyurek, E. et al. Broadband waveguide quantum memory for entangled photons. Nature 469, 512-515 (2011).

21. Zhong, M. et al. Optically addressable nuclear spins in a solid with a six-hour coherence time. Nature 517, 177-180 (2015).

22. Gao, W. B. et al. Observation of entanglement between a quantum dot spin and a single photon. Nature 491, 426-430 (2012).

23. Chou, C. W. et al. Measurement-induced entanglement for excitation stored in remote atomic ensembles. Nature 438, 828-832 (2005).

24. Yuan, Z.-S. et al. Experimental demonstration of a BDCZ quantum repeater node. Nature 454, 1098-1101 (2008).

25. Matsukevich, D. N. et al. Entanglement of remote atomic qubits. Phys. Rev. Lett. 96, 030405 (2006).

26. Choi, K. S., Deng, H., Laurat, J. \& Kimble, H. J. Mapping photonic entanglement into and out of a quantum memory. Nature 452, 67-71 (2008)

27. Zhang, W. et al. Experimental realization of entanglement in multiple degrees of freedom between two quantum memories. Nat. Commun. 7, 13514 (2016).

28. Choi, K. S., Goban, A., Papp, S. B., Van Enk, S. J. \& Kimble, H. J. Entanglement of spin waves among four quantum memories. Nature 468, 412-416 (2010).

29. Julsgaard, B., Kozhekin, A. E. \& Polzik, E. S. Experimental long-lived entanglement of two macroscopic objects. Nature 413, 400-403 (2001).

30. Krauter, H. et al. Entanglement generated by dissipation and steady state entanglement of two macroscopic objects. Phys. Rev. Lett. 107, 080503 (2011)

31. Ladd, T. D. et al. Quantum computers. Nature 464, 45-53 (2010).

32. Sangouard, N., Simon, C., Riedmatten, H. D. \& Gisin, N. Quantum repeaters based on atomic ensembles and linear optics. Rev. Mod. Phys. 83, 33-80 (2011).

33. Yadsanappleby, H. \& Serafini, A. Would one rather store squeezing or entanglement in continuous variable quantum memories. Phys. Lett. A 375, 1864-1869 (2011).

34. Tikhonov, K. S., Golubeva, T. Y. \& Golubev, Y. M. Entangled states of signal pulses in multimode quantum memory. Opt. Spectrosc. 118, 773-780 (2015).

35. Jensen, $\mathrm{K}$. et al. Quantum memory for entangled continuous-variable states. Nat. Phys. 7, 13-16 (2011).

36. Bachor, H. A. \& Ralph, T. C. A Guide to Experiments in Quantum Optics 2nd edn (Wiley-VCH, 2004).

37. $\mathrm{Su}, \mathrm{X}$. L. et al. Experimental preparation of eight-partite cluster state for photonic qumodes. Opt. Lett. 37, 5178-5180 (2012).

38. Jia, X. J. et al. Superactivation of multipartite unlockable bound entanglement. Phys. Rev. Lett. 108, 190501 (2012).

39. Jia, X. et al. Experimental realization of three-color entanglement at optical fiber communication and atomic storage wavelengths. Phys. Rev. Lett. 109, 253604 (2012).

40. Wu, L. et al. Experimental generation of tripartite polarization entangled states of bright optical beams. Appl. Phys. Lett. 108, 161102 (2016).

41. Fleischhauer, M., Imamoglu, A. \& Marangos, J. P. Electromagnetically induced transparency: optics in coherent media. Rev. Mod. Phys. 77, 633-673 (2005).

42. Fleischhauer, M. \& Lukin, M. D. Dark-state polaritons in electromagnetically induced transparency. Phys. Rev. Lett. 84, 5094-5097 (2000). 
43. Cviklinski, J. et al. Reversible quantum interface for tunable single-sideband modulation. Phys. Rev. Lett. 101, 133601 (2008).

44. Appel, J., Figueroa, E., Korystov, D., Lobino, M. \& Lvovsky, A. I. Quantum memory for squeezed light. Phys. Rev. Lett. 100, 093602 (2008).

45. Honda, K. et al. Storage and retrieval of a squeezed vacuum. Phys. Rev. Lett. 100, 093601 (2008).

46. Ou, Z. Y. Efficient conversion between photons and between photon and atom by stimulated emission. Phys. Rev. A 78, 023819 (2008).

47. He, Q. Y., Reid, M. D., Giacobino, E., Cviklinski, J. \& Drummond, P. D. Dynamical oscillator-cavity model for quantum memories. Phys. Rev. A 79, 022310 (2009).

48. Van Loock, P. \& Furusawa, A. Detecting genuine multipartite continuousvariable entanglement. Phys. Rev. A 67, 052315 (2003).

49. Ou, Z. Y., Pereira, S. F., Kimble, H. J. \& Peng, K. C. Realization of the Einstein-Podolsky-Rosen Paradox for continuous variables. Phys. Rev. Lett. 68, 3663 (1992).

50. Yokoyama, S. et al. Ultra-large-scale continuous-variable cluster states multiplexed in the time domain. Nat. Photon 7, 982-986 (2013).

51. Roslund, J., Medeiros, R., Jiang, S., Fabre, C. \& Treps, N. Wavelengthmultiplexed quantum networks with ultrafast frequency combs. Nat. Photon $\mathbf{8}$, 109-112 (2014).

52. Phillips, N. B., Gorshkov, A. V. \& Novikova, I. Light storage in an optically thick atomic ensemble under conditions of electromagnetically induced transparency and four-wave mixing. Phys. Rev. A 83, 063823 (2011).

53. Lobino, M., Kupchak, C., Figueroa, E. \& Lvovsky, A. I. Memory for light as a quantum process. Phys. Rev. Lett. 102, 203601 (2009).

54. Lauk, N., O'Brien, C. \& Fleischhauer, M. Fidelity of photon propagation in electromagnetically induced transparency in the presence of four-wave mixing. Phys. Rev. A 88, 013823 (2013).

55. Yang, S.-J., Wang, X.-J., Bao, X.-H. \& Pan, J.-W. An efficient quantum light--matter interface with sub-second lifetime. Nat. Photon 10, 381-384 (2016).

56. Saunders, D. J. et al. Cavity-enhanced room-temperature broadband Raman memory. Phys. Rev. Lett. 116, 090501 (2016).

57. Vahlbruch, H., Mehmet, M., Danzmann, K. \& Schnabel, R. Detection of $15 \mathrm{~dB}$ squeezed states of light and their application for the absolute calibration of photoelectric quantum efficiency. Phys. Rev. Lett. 117, 110801 (2016).

58. Barrett, S. D., Rohde, P. P. \& Stace, T. M. Scalable quantum computing with atomic ensembles. New J. Phys. 12, 093032 (2010).

\section{Acknowledgements}

The authors acknowledge financial support from the Key Project of the Ministry of Science and Technology of China (Grant No. 2016YFA0301402), the Natural Science Foundation of China (Grant Nos. 61775127, 11474190, 11654002), FOK YING TUNG Education Foundation, the Program for Sanjin Scholars of Shanxi Province.

\section{Author contributions}

X.J., H.W., and C.X. conceived the original idea. Z.Y., L.W., X.J., and K.P. designed the experiment. Z.Y., L.W., Y.L., and R.D. constructed and performed the experiment. Z.Y., X.J., and S.L. accomplished theoretical calculation and the data analysis. Z.Y., X.J., C.X and K.P. wrote the paper. All the authors reviewed the manuscript.

\section{Additional information}

Competing interests: The authors declare no competing financial interests.

Reprints and permission information is available online at http://npg.nature.com/ reprintsandpermissions/

Publisher's note: Springer Nature remains neutral with regard to jurisdictional claims in published maps and institutional affiliations.

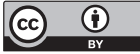

Open Access This article is licensed under a Creative Commons Attribution 4.0 International License, which permits use, sharing, adaptation, distribution and reproduction in any medium or format, as long as you give appropriate credit to the original author(s) and the source, provide a link to the Creative Commons license, and indicate if changes were made. The images or other third party material in this article are included in the article's Creative Commons license, unless indicated otherwise in a credit line to the material. If material is not included in the article's Creative Commons license and your intended use is not permitted by statutory regulation or exceeds the permitted use, you will need to obtain permission directly from the copyright holder. To view a copy of this license, visit http://creativecommons.org/ licenses/by/4.0/

(C) The Author(s) 2017 